\title{
Impulse control and related behaviors in Parkinson's disease with dementia
}

Pablo Martinez-Martin, MD, $\mathrm{PhD}^{1}$, Yi Min Wan, $\mathrm{MD}^{2,3,4}$, K. Ray Chaudhuri, MD ${ }^{2,3}$; Anette E. Schrag, MD ${ }^{5}$, Daniel Weintraub, $\mathrm{MD}^{6,7}$

${ }^{1}$ National Center of Epidemiology and CIBERNED. Carlos III Institute of Health, Madrid, Spain

${ }^{2}$ Institute of Psychiatry, Psychology \& Neuroscience at King's College London, Department of Basic \& Clinical Neuroscience, De Crespigny Park, London, SE5 8AF, United Kingdom

${ }^{3}$ Parkinson Foundation Centre of Excellence at King's College Hospital, Denmark Hill, London, SE5 9RS, United Kingdom

${ }^{4}$ Department of Psychiatry, Ng Teng Fong General Hospital, Singapore 609606.

${ }^{5}$ Department of Clinical Neurosciences, Royal Free Campus UCL Institute of Neurology, UK

${ }^{6}$ Departments of Psychiatry and Neurology, Perelman School of Medicine at the University of Pennsylvania, Philadelphia, USA

${ }^{7}$ Parkinson's Disease Research, Education and Clinical Center (PADRECC), Philadelphia Veterans Affairs Medical Center, Philadelphia, USA

\section{Corresponding author:}

Yi Min Wan, MD

Department of Psychiatry,

$\mathrm{Ng}$ Teng Fong General Hospital

1 Jurong East Street 21,

Singapore 609606.

Tel: +6567162000

Fax: +6567165500

E-mail: yi min.wan@kcl.ac.uk 


\section{Manuscript details:}

Abstract word count -173

Manuscript word count- 4018

Number tables/figures -2 tables, 2 supplementary tables

References - 27

Running title: Impulse Control and related behaviors in Parkinson's Disease Dementia

Key words: Parkinson's disease, dementia, punding, hobbyism, impulse control disorder, cognitive impairment, dopamine agonists, dopamine dysregulation syndrome

Conflicts of Interest: The authors declare that there are no conflicts of interest relevant to this work.

\section{Financial Disclosures of all authors (for the preceding 12 months)}

PMM: Honoraria: from National School of Public Health (ISCIII) and Editorial Viguera for lecturing in courses; International Parkinson and Movement Disorder Society (IPMDS) for management of the Program on Rating Scales; Air Liquide, Abbvie, Zambon, and HM Hospitales de Madrid for advice in clinical-epidemiological studies. License fee payments for the King's Parkinson's Disease Pain scale. Grant of the IPMDS for attending the Congress of 2018 and research grant for development and validation of the MDS-NMS.

YMW: None

KRC: Advisory board: AbbVie, UCB , Sunovion, Pfizer, Jazz Pharma, GKC, Bial, Cynapsus, Novartis, Lobsor, Stada, Medtronic, Zambon, Profile. Honoraria for lectures: AbbVie ,Britannia, UCB, Mundipharma, Zambon, Novartis, Boeringer Ingelheim Neuroderm, Sunovion. Grants (Investigator Initiated) : Britannia Pharmaceuticals, AbbVie, UCB, GKC, Bial; Aacdemic grants: EU, IMI EU, Horizon 2020, Parkinson's UK, NIHR, PDNMG, Kirby Laing Foundation, NPF, MRC UK, NMRC Singapore. 
AS: Advisory Board: Medtronic, Roche, Biogen; Grant: EU Commission, Parkinson's UK, GE Healthcare, Economic and Social Research Council (ESRC), International Parkinson's and Movement Disorders Society, National Institute of Health (NIHR), National Institute for Health Research ULCH Biomedical Research.

DW: Received research funding or support from Michael J. Fox Foundation for Parkinson's Research, National Institutes of Health (NINDS), Department of Veterans Affairs, Alzheimer's Therapeutic Research Initiative (ATRI), Alzheimer's Disease Cooperative Study (ADCS), and the International Parkinson and Movement Disorder Society (IPMDS); honoraria for consultancy from Acadia, Alkahest, CHDI Foundation, Clintrex LLC, F. Hoffmann-La Roche Ltd and Sunovion; license fee payments from the University of Pennsylvania for the QUIP and QUIP-RS. 


\begin{abstract}
Background: The study objective was to determine the frequency, demographic and clinical correlates (such as age, sex, PD severity, and dopaminergic treatment) of impulse control disorder symptoms (ICDs) and related behaviors in Parkinson's disease (PD) patients with (PD-D) and without (PD-ND) dementia.
\end{abstract}

Methods: We analyzed historical data from a national, multi-center, cross-sectional database, and assessed ICDs and related behaviors with the Scale for Evaluation of Neuropsychiatric Disorders in PD (SEND-PD) administered as a semi-structured interview to PD-D (N=85) and PD-ND (N=444) patients, and their informants.

Results: Dopamine agonist therapy use was common and comparable in the two groups $(78.8 \%$ in PD-D vs. $82.9 \%$ in PD-ND), but ICDs (23.5\% vs. $13.3 \%$, p=0.02), hobbyism-punding (32.9\% vs. $10.6 \%, \mathrm{p}<0.001)$ and dopaminergic medication abuse $(8.2 \%$ vs. $3.2 \%, \mathrm{p}=0.03)$ were more common in the PD-D group.

Conclusions: The finding that ICDs and related behaviors are more common in PD patients usually treated with dopamine agonists who also have comorbid dementia, suggests that the neural substrates associated with PD dementia may also predispose to development of compulsive behaviors. 


\section{INTRODUCTION}

Impulse control disorder (ICDs) and related behaviors, including compulsive gambling, buying, sexual, and eating behaviors, hobbyism, punding, and dopamine dysregulation syndrome, can occur as a behavioral complication of dopamine replacement therapy (DRT), particularly with dopamine agonists (DAs) but also with higher-dose levodopa, in patients with Parkinson's disease (PD)(1-3). Additional variables associated with ICDs in different studies include types of DA, younger age, male sex and early onset of PD (4-6).

As the strongest risk factors for ICD development in PD are DA treatment and younger age, and given that cognitively impaired patients are typically older and less likely prescribed a DA, it is not surprising that ICDs have not been commonly reported in PD patients with comorbid dementia (PDD). In Poletti et al.'s 2013 cross-sectional single-center study of 805 PD patients, ICD symptoms were less common in PD-D patients compared with non-demented patients (PD-ND) (3.8\% vs. 9.6\%), but only $16.4 \%$ of the PD-D patients were on a DA compared with $63.2 \%$ of the PD-ND group. As such, this may have been an artifact of DA prescribing practice (7).

Here we utilize a large multicenter PD database, including 67 demented patients prescribed a DA, to examine the frequency and correlates of ICDs in PD patients with and without dementia. Our hypothesis was that the rates of ICDs would be lower among PD-D as compared with PD-ND patients, consistent with the different rates of DA prescription between both groups, as well as the literature to date.

\section{METHODS}

\section{Design}

This study used data from a large multicenter, cross-sectional, observational, nationwide (Spain) psychometric scale validation study in PD (8), collected from 2010 to 2011.

\section{Patients}


Consecutive patients were included if they met the following criteria: [1] diagnosis of PD by a neurologist applying PD-validated diagnostic criteria (9); [2] participating primary caregiver; and [3] signed informed consent for study participation (8). Patients who did not meet all inclusion criteria and those with a severe disease or health condition that could interfere with the appropriate assessment of PD manifestations were excluded.

\section{Caregivers}

The primary caregiver was defined as "any person who, without being a professional or belonging to a social support network, usually lives with the patient and in some way is directly implicated in the patient's care or is directly affected by the patient's health problem" (10).

\section{Assessments}

We administered the following instruments:

1. Hoehn and Yahr staging (HY) for disease severity (11).

2. Scales for Outcomes in Parkinson's Disease-Motor (SCOPA-Motor) $(12,13)$.

3. Mini-Mental State Examination (MMSE) for global cognition (14).

4. Lexical fluency (score $\leq 9$ considered as impairment) (15).

5. The Pill Questionnaire, an index of mental organization and functioning in a daily living situation (16).

6. Clinical Impression of Severity Index-PD (CISI-PD), a global evaluation based on the clinical impression of overall PD severity.(17, 18).

7. Scale for Evaluation of Neuropsychiatric Disorders in PD (SEND-PD) (8), a health professionalbased assessment scored through an interview with the patient or caregiver. It is composed of 12 items, each one scoring for severity from 0 (not present) to 4 (very severe), divided into three subscales: psychotic symptoms (4 items), mood/apathy (5 items), and ICDs and related behaviors (3 items). The latter three items cover: (i) hobbyism-punding, phrased as, "Does he/she undertake repetitive activities daily without any real need, spend a long time doing it, or feel annoyed and irritated if not allowed or recriminated for it?; (ii) ICDs, phrased as, "Has he/she presented with 
impulsive behavior, things he/she cannot stop doing, such as: doing excessive or unnecessary shopping, spending lots of time and money in gambling, or exaggerated sexual interest?"; and (iii) dopaminergic drug abuse (i.e., dopamine dysregulation syndrome), phrased as, "Does he/she take medication with a higher frequency or dose than prescribed?". The SEND-PD has been validated in three separate studies $(8,19,20)$.

\section{Procedure}

Data were captured by 106 neurologists in a Spanish nationwide study from April 2010 until May 2011(8). We administered the interview primarily to the patient in the presence of the caregiver, who could correct, clarify, or provide the responses in case of patient unawareness or unreliability. An independent company (Pivotal S.L.) monitored the study. Once collected and properly recorded, data were transferred to an author (PMM) for data analysis.

Patients were categorized into probable PD-D or PD-ND (21). Diagnosis of PD-D was operationalized as: [1] PD according to recognized diagnostic criteria (9); [2] dementia syndrome developed at least 1 year after PD onset (21); [3] MMSE score <26 (15); [4] impairment in at least two of the following tests: serial sevens, lexical fluency, MMSE pentagons, and 3-word recall (15); [5] Pill Questionnaire showing impact on ADLs (16); and [6] absence of severe depressive and psychotic manifestations (score $\leq 2$ in the SEND-PD items for depression, hallucinations, and delusions) and absence of delirium $(15,21)$

\section{Ethical aspects}

The scale validation study, from which data for this present one are derived, was approved by the Ethics Committee of the Alzheimer Disease Research Unit, CIEN Foundation, Carlos III Institute of Health, and was reported to the Spanish Medicines Agency (Protocol code: 06-NEU-ANEP-01-0110). Written information about the study was provided to the participant candidates and their caregivers and was complemented with verbal information by the researcher if required. The 
information material and signature documents were reviewed and accepted by the Ethics Committee. All patients and caregivers signed the informed consent for participation in the study.

\section{Data analysis}

The SEND-PD evaluates the severity of the neuropsychiatric manifestations, but the prevalence of the neuropsychiatric symptoms can also be determined considering SEND-PD items as absent (score=0) or present (score $\geq 1$ ). In addition to descriptive statistics for SEND-PD, the following methods were used, as needed: [1] calculation of the levodopa equivalent daily dose (LEDD) (22); a separate calculation was carried out for DA and total (DA plus levodopa) LEDD; [2] as main data were ordinal or did not fit normal distribution (Shapiro-Francia test; all p <0.0001), the Mann-Whitney or KruskalWallis test were used for comparison between groups; [3] bivariate associations were analyzed with the Spearman rank correlation coefficient; values $>0.60$ were considered a "strong correlation"; and [4] multiple logistic regression models for detecting predictors of presence of the neuropsychiatric disorders were constructed using sets of independent variables without relevant collinearity among them (all with Spearman correlation coefficients $<0.60$ and postestimation variance inflation factors $1 / \mathrm{VIF}>0.60$ ). Non-parametric tests were used for comparison and correlation because the non-normal distribution of the main variables (Shapiro-Francia test; all $<0.0001$ ). For multiple testing correction balanced for type I and type II errors, the Benjamini-Hochberg method was applied (23). Comparison of correlation coefficients was carried out by Fisher z transformation.

\section{RESULTS}

\section{Participant characteristics}

Six hundred thirty-three patients were included in the original study (8). Missing data, incomplete cognitive state evaluation, or any other problem to make a clear diagnosis of PD-D or PD-ND led to exclusion of 104 patients (16.43\%). Excluded patients were older ( $\mathrm{p}=0.005)$, had more severe motor disorder $(\mathrm{p}<0.001)$ and had more advanced disease $(\mathrm{p}<0.001)$ than included patients. Their DA daily dose was significantly lower than for included patients ( $209.86 \pm 157.30$ vs. $261.70 \pm 165.56 \mathrm{mg} /$ day; $\mathrm{p}<0.0001)$. 


\section{Frequency and correlates of dementia}

Eighty-five of $529(16.1 \%)$ patients were diagnosed with PD-D; characteristics of the sample by dementia status are in Table 1. PD-D patients, compared with PD-ND patients, were older, had longer duration and later onset of $\mathrm{PD}$, and were more impaired on all motor and non-motor measures. DA use was common and similar in both groups (78.8\% of PD-D vs. $82.8 \%$ of PD-ND, p=0.38) and DA LEDD was similar ( $\mathrm{p}=0.19)$. However, we note that the higher total LEDD in the PD-D group, in the face of similar DA LEDD, reflected that higher doses of levodopa had been used in the PD-D group.

\section{Prevalence and severity of ICD and related behavior symptoms by dementia status}

The prevalence and severity of the ICDs are shown in Table 2. After correction for multiple comparisons, ICDs and hobbyism-punding were significantly more common in PD-D compared with PD-ND patients. In addition, the mean severity of SEND-PD scores was higher in PD-D patients for all ICDs and related behaviors. The rates and severity of dopaminergic drug abuse were also significantly higher in PD-D patients than in PD-ND.

In those treated with a DA, impulse control disorder and hobbyism-punding symptoms were more common compared with non-DA-treated patients (Supplementary Table 1). Examining rates of behaviors in DA-treated patients only, they were 2-3 times higher in patients with co-morbid dementia vs. those without dementia. The sample size of patients not on a DA was too small to do a meaningful between-group comparison.

\section{Multi-variable predictors of ICD or related behavior disorder symptoms}

Examining the entire study population, in two multiple logistic regression models (one with DA LEDD and the other with total LEDD included as a co-variate), co-morbid dementia was independently associated with presence of hobbyism/punding in both models (Supplementary Table 2). In addition, increasing disease severity in both models was an independent predictor of all three 
behaviors; younger age predicted ICDs, and hobbyism-punding behaviors, and male sex predicted presence of ICDs.

\section{DISCUSSION}

In this examination of ICDs and related behaviors in a cross-sectional, national, multi-site PD cohort with very high dopamine agonist use from 2010 until 2011, we found that the symptoms of ICDs, hobbyism-punding, and dopaminergic drug abuse were more common and more severe in demented compared with non-demented patients. Consistent with previous research, DA use was associated with both ICDs and hobbyism-punding behaviors. In multi-variable models, other predictors of some or all behaviors, also consistent with previous research, were increasing disease severity, younger age and male sex.

We also found that patients with comorbid dementia had higher rates of all three behavioral variants compared with their non-demented counterparts, both in the DA-treated group and in the overall population. In an aforementioned study (7), ICD rates were lower in PD-D compared with PD-ND patients, but this may have been because demented patients were not commonly prescribed a DA or higher doses of levodopa therapy. The association we observed between dementia and higher rates of ICDs and related behaviors in PD has not been reported previously to our knowledge, likely in part because of the fact that DAs, the primary medication risk factor for ICD development, are not commonly prescribed to older patients or those with dementia, due to concerns about side effects including psychosis, confusion and sedation. However, in this population, nearly $80 \%$ of dementia patients were taking a DA, comparable to non-demented patients. The findings suggest that in the face of DA treatment, comorbid dementia may likely increase the risk of developing ICDs and related behaviors, although causality cannot be determined from a cross-sectional study. It is important to note that higher total levodopa exposure may also have contributed to the higher frequency of ICD behaviors in the PD-D group, consistent with previous research from the DOMINION study that suggested higher levodopa exposure was associated with ICDs in PD (6). However, even when 
accounting for this in a multivariate analysis, PD dementia still significantly contributed to prediction of hobbyism-punding.

The underlying cause of the increased rate of ICDs in patients with PD-D is unclear. However, an increased rate of neuropsychiatric symptoms, such as psychosis and REM sleep behavior disorder, is well-recognized in PD-D, likely reflecting the more widespread distribution of PD-related pathology in brain areas involved in emotional and behavioral functioning, and more extensive involvement of non-dopaminergic neurotransmitter dysfunction. It is also possible that an increase in ICD-like behaviors captured in the study questions is a non-specific phenomenon in patients with dementia. Disinhibition is common in Alzheimer's disease (AD) and increased even in patients with mild cognitive impairment (24). However, these studies used the Neuropsychiatric Inventory to assess symptomatology, which does not specifically assess ICDs, and ICD-type behaviors have not been specifically reported in other dementias such as AD.

Whilst compulsive behaviors such as hyper-orality are common in patients with fronto-temporal dementia (FTD), the presentation of these behaviors are different from ICDs in PD, and the underlying neuropathology of FTD differs significantly to that of PD-D. In addition, more complex, repetitive behavioral routines are also common in FTD, reported to occur in $22 \%$ of patients (25), and it is possible that PD-D itself might manifest such frontally-mediated signs. Bentivoglio and coworkers suggested a frontal lobe dysfunction, with imbalance between ventral and dorsal frontostriatal loops, in patients with ICDs (26). However, to date repetitive behaviors mimicking ICDs and related behaviors have not clearly been associated with PD-D specifically.

In terms of limitations, our study design using the MMSE did not allow us to determine if frontal executive deficits are primarily associated with ICDs and repetitive behaviors in this population. 
features in PD-D and other dementias, including instruments that assess ICD-like behaviors such as the SEND-PD, and assess specific cognitive domain in more detail.

In addition, we note that DA prescribing was much higher across the PD sample population, in particular among the PD-D patients, than is considered advisable in updated clinical practice guidelines. However, data for this study was obtained in 2010 and 2011, a period during which DA agonist use was much higher among PD patients in general compared with now(27), in part due to the limited data on ICDs in PD at that time. Finally, the SEND-PD includes only three questions to evaluate ICDs and related behaviors, does not include compulsive eating, and this section of the SEND-PD has not been validated. However, there were no PD-specific ICD rating scales available when this data was collected.

Our results demonstrate that ICDs and related behaviors are at least as common, if not more common, in primarily DA-treated PD patients with comorbid dementia compared with non-demented patients, suggesting that the neural substrates underpinning dementia in PD may predispose to the development of compulsive behaviors in this population under DA treatment. If so, this could be related to dementia-associated monoaminergic deficits, changes in neural pathways such as cortical (e.g., prefrontal cortex) deficits or cortical-striatal connectivity changes, or cognitive impairments. Regardless, the findings of this study demonstrate that significant cognitive impairment may be a risk factor for development of ICDs and related behaviors when biases in prescription patterns are accounted for, which is of clinical significance and provides additional justification for using DAs cautiously in patients with significant cognitive impairment.

\section{Table 1}

\section{Table 2}




\section{Supplementary Table 1}

\section{Supplementary Table 2}

\section{Acknowledgments}

The authors acknowledge the contribution of the neurologists listed in the Appendix.

\section{Funding Sources:}

The study from which data for this research are derived was funded by UCB Pharma, Madrid, Spain. Pivotal SL oversaw collection of the data, which were provided by the neurologists listed in the Appendix.

\section{Conflicts of Interest:}

The authors declare that there are no conflicts of interest relevant to this work.

\section{Appendix}

M Aguado Valcarce; E Agüera Morales; M Álvarez Sauco; JM Arbelo González; M Arias Rodríguez; J Artal Roy; A Ávila; J Balseiro; B Berlanga Morán; M Blázquez Estrada; L Brieva Ruiz; M Bujanda Alegría; I Cabo; A Callén Soto; V Campos Arillo; JA Cárdenas Rodulfo; J Carod; P Casariego; C Castejón Gabriel; MJ Catalán; MÁ Ceballos; E Cebrián; F Coria Balanzat; E Cubo Delgado; T Delgado Ballestero; AM Espino Ibáñez; R Espinosa Rosso; O Fernández Arconada; V Fernández 
Armayor; Á Fernández Díaz; JM Fernández García; JJ Flores Ruiz; ML Galiano Fragua LS García Andrade; L García Fernández; V García Gil; S García Muñoguren; MJ Garea García-Malvar; A Gil Rodrigo; D Gómez; MJ Gómez Heredia; J Ma Gómez Reino; C González Oria; V González Torres; L Gubieras Lillo; J Gutiérrez García ; JJ Hernández Regader; EE Hernández Valero; S Huertas Folch; M Huertas Villanueva; B Indakoetxea; J Juni Sanahuja; M Lara Lara; S López Calvo; JM López Domínguez; L Lopez Manzanares; JN Lorenzo Brito; M Llaneza González; MJ Magraner Benedicto; A Malagelada Seckler; J Marey López; S Martí Martínez; S Martín Balbuena; JL Martín Gurpegui; J Martín Polo; A Martínez Gil; I Martinez Torres; V Mateos Marcos; S Mederer; V Medrano; M Menéndez González; P Millet Balanzo; A Mínguez Castellanos; P Modrego Pardo; A Montalbo; O Morsi Hassan; M Moya Molina; M Ochoa; J Olivares Romero; N Ortíz Castellón; A Ortiz Pascual; AM Pascual Lozano; A Pato Pato; R Pego Reigosa; F Pérez Errazquin; V Peset Mancebo; J Prat Rojo; N Pérez; JL Pérez López; MC Pérez Viéitez; A Ponz de Tienda; JM Ramírez Moreno; C Ricart Colomé; A Robledo Strauss; I Rodríguez; N Rodríguez Fernández; A Rojo; J Ruiz Martínez; J Salmero Martínez; A Salvador Aliaga; O Sanchez del Valle; A Sesar Ignacio; MD Sevillano; G Soriano Hernández; A Vadillo Bermejo; and F Vivancos Matellanos. 


\section{REFERENCES}

1. Weintraub D, Nirenberg MJ. Impulse control and related disorders in Parkinson's disease. Neurodegener Dis. 2013;11(2):63-71.

2. Giovannoni G, O'Sullivan JD, Turner K, Manson AJ, Lees AJ. Hedonistic homeostatic dysregulation in patients with Parkinson's disease on dopamine replacement therapies. J Neurol Neurosurg Psychiatry. 2000;68(4):423-8.

3. Evans AH, Katzenschlager R, Paviour D, O'Sullivan JD, Appel S, Lawrence AD, et al. Punding in Parkinson's disease: its relation to the dopamine dysregulation syndrome. Mov Disord. 2004;19(4):397-405.

4. Rizos A, Sauerbier A, Antonini A, Weintraub D, Martinez-Martin P, Kessel B, et al. A European multicentre survey of impulse control behaviours in Parkinson's disease patients treated with short- and long-acting dopamine agonists. Eur J Neurol. 2016;23(8):1255-61.

5. Voon V, Fox SH. Medication-related impulse control and repetitive behaviors in Parkinson disease. Arch Neurol. 2007;64(8):1089-96.

6. Weintraub D, Koester J, Potenza MN, Siderowf AD, Stacy M, Voon V, et al. Impulse control disorders in Parkinson disease: a cross-sectional study of 3090 patients. Arch Neurol. 2010;67(5):58995.

7. Poletti M, Logi C, Lucetti C, Del Dotto P, Baldacci F, Vergallo A, et al. A single-center, cross-sectional prevalence study of impulse control disorders in Parkinson disease: association with dopaminergic drugs. J Clin Psychopharmacol. 2013;33(5):691-4.

8. Martinez-Martin P, Frades-Payo B, Aguera-Ortiz L, Ayuga-Martinez A. A short scale for evaluation of neuropsychiatric disorders in Parkinson's disease: first psychometric approach. J Neurol. 2012;259(11):2299-308.

9. Lees AJ, Hardy J, Revesz T. Parkinson's disease. Lancet. 2009;373(9680):2055-66.

10. Martinez-Martin P, Forjaz MJ, Frades-Payo B, Rusinol AB, Fernandez-Garcia JM, BenitoLeon J, et al. Caregiver burden in Parkinson's disease. Mov Disord. 2007;22(7):924-31; quiz 1060. 
11. Hoehn MM, Yahr MD. Parkinsonism: onset, progression and mortality. Neurology. 1967;17(5):427-42.

12. Marinus J, Visser M, Stiggelbout AM, Rabey JM, Martinez-Martin P, Bonuccelli U, et al. A short scale for the assessment of motor impairments and disabilities in Parkinson's disease: the SPES/SCOPA. J Neurol Neurosurg Psychiatry. 2004;75(3):388-95.

13. Martinez-Martin P, Benito-Leon J, Burguera JA, Castro A, Linazasoro G, Martinez-Castrillo JC, et al. The SCOPA-Motor Scale for assessment of Parkinson's disease is a consistent and valid measure. J Clin Epidemiol. 2005;58(7):674-9.

14. Folstein MF, Folstein SE, McHugh PR. "Mini-mental state". A practical method for grading the cognitive state of patients for the clinician. J Psychiatr Res. 1975;12(3):189-98.

15. Dubois B, Burn D, Goetz C, Aarsland D, Brown RG, Broe GA, et al. Diagnostic procedures for Parkinson's disease dementia: recommendations from the movement disorder society task force. Mov Disord. 2007;22(16):2314-24.

16. Martinez-Martin P. Dementia in Parkinson's disease: usefulness of the pill questionnaire. Mov Disord. 2013;28(13):1832-7.

17. Martinez-Martin P, Forjaz MJ, Cubo E, Frades B, de Pedro Cuesta J, Members EP. Global versus factor-related impression of severity in Parkinson's disease: a new clinimetric index (CISI-PD). Mov Disord. 2006;21(2):208-14.

18. Martinez-Martin P, Rodriguez-Blazquez C, Forjaz MJ, de Pedro J, Spanish-American Longitudinal PDPSG. The Clinical Impression of Severity Index for Parkinson's Disease: international validation study. Mov Disord. 2009;24(2):211-7.

19. Meyer A, Hadinia A, Hatz F, Martinez-Martin P, Fuhr P, Stieglitz RD, et al. [German Translation and Validation of the Scale for Evaluation of Neuropsychiatric Disorders in Parkinson's disease (SEND-PD)]. Fortschr Neurol Psychiatr. 2016;84(7):421-7.

20. Rodriguez-Violante M, Cervantes-Arriaga A, Velazquez-Osuna S, Llorens-Arenas R, Calderon-Fajardo H, Pina-Fuentes D, et al. Independent Validation of the SEND-PD and Correlation with the MDS-UPDRS Part IA. Parkinsons Dis. 2014;2014:260485. 
21. Emre M, Aarsland D, Brown R, Burn DJ, Duyckaerts C, Mizuno Y, et al. Clinical diagnostic criteria for dementia associated with Parkinson's disease. Mov Disord. 2007;22(12):1689-707; quiz 837.

22. Tomlinson CL, Stowe R, Patel S, Rick C, Gray R, Clarke CE. Systematic review of levodopa dose equivalency reporting in Parkinson's disease. Mov Disord. 2010;25(15):2649-53.

23. Benjamini YH, Y. Controlling the false discovery rate: A Practical and powerful approach to multiple testing. Journal of the Royal Statistical Society. 1995;57(1):289-300.

24. Lyketsos CG, Lopez O, Jones B, Fitzpatrick AL, Breitner J, DeKosky S. Prevalence of neuropsychiatric symptoms in dementia and mild cognitive impairment: results from the cardiovascular health study. JAMA. 2002;288(12):1475-83.

25. Snowden JS, Bathgate D, Varma A, Blackshaw A, Gibbons ZC, Neary D. Distinct behavioural profiles in frontotemporal dementia and semantic dementia. J Neurol Neurosurg Psychiatry. 2001;70(3):323-32.

26. Bentivoglio AR, Baldonero E, Ricciardi L, De Nigris F, Daniele A. Neuropsychological features of patients with Parkinson's disease and impulse control disorders. Neurol Sci. 2013;34(7):1207-13.

27. Pellicano C, Benincasa D, Fanciulli A, Latino P, Giovannelli M, Pontieri FE. The impact of extended release dopamine agonists on prescribing patterns for therapy of early Parkinson's disease: an observational study. Eur J Med Res. 2013;18:60. 